\title{
Logicality as Necessary Instantiation
}

[Logicalidade como Instanciação Necessária]

\author{
Roderick Batchelor ${ }^{*}$
}

\begin{abstract}
We propose to define logicality of a simple notion as necessary instantiation. (And then logicality of a complex notion as logicality of all simple notions occurring in it.)

Keywords: logicality, logical constants, necessary existence, necessary instantiation.

Resumo: Propomos definir logicalidade de uma noção simples como instanciação necessária. (E então logicalidade de uma noção complexa como logicalidade de todas as noções simples nela ocorrendo.)

Palavras-chave: logicalidade, constantes lógicas, existência necessária, instanciação necessária.
\end{abstract}

\section{Introduction}

There seems to be a close link between logicality and necessity; and correlatively a close link between empiricality and contingency. Dramatically speaking, the logical realm is the realm of necessities, whereas the empirical realm is the realm of contingencies. (I mean logicality here in a broad sense; some might prefer to say 'logico-mathematicality'.)

The very existence of things or of instances of kinds of things is, typically, contingent in empirical cases and necessary in logical cases. Thus a paradigmatic empirical thing like the Eiffel Tower might of course not have existed; and a paradigmatic empirical concept like the concept of dog might not have had any instances. But a paradigmatic logical (or if you like logico-mathematical) thing like the empty set is no doubt a necessary existent (at least if it exists at all), and a paradigmatic logical concept like identity is necessarily instantiated.

One might then think of defining logicality as something like this - necessary existence or necessary instantiation. However, there are obvious counterexamples. The concept [dog or nondog] is necessarily instantiated, but not logical; and the concept [identical and non-identical with] is logical, but not necessarily instantiated (indeed necessarily uninstantiated).

But in such counterexamples we artfully construct certain com-

${ }^{\star}$ Lecturer in Philosophy at the University of São Paulo. E-mail: roderick_batchelor@hotmail.com. 
plex concepts. Perhaps we can still use the idea above to define logicality for simples, and then somehow extend the definition to complexes? - I will argue in what follows that indeed we can.

\section{A definition of logicality}

By an attributive notion I mean a notion which may be 'attributed' or 'applied' to other notions so as to form a proposition. Possible examples of attributive notions are: the notions expressed by ordinary verbal expressions like 'smokes' or 'is parallel to'; propositional 'connectives', such as the notions expressed by 'and', 'Socrates believes that', etc.; quantifiers and other notions which produce propositions upon application to notions which are themselves attributive - thus [Something], [Every philosopher], etc.

By a substance-notion I mean a notion which is not attributive. Thus e.g. the notions expressed by 'Socrates' and by 'All men are mortal' are substance-notions.

I say that a notion is complex if at least one other notion enters into its constitution - thus e.g. [white horse] is of course a complex notion. And I say that a notion is simple if it is not complex.

By saying that an attributive notion is instantiated I mean that there is some true proposition consisting of the attributive notion in question applied to some notion or notions. Thus [white horse] is instantiated, but [flying horse] is not.

I will use also the concept of the referent of a simple substancenotion. (Different philosophers may wish to define this in different ways. I do not exclude that the referent be identified with the simple substance-notion itself.) By saying that a simple substancenotion is instantiated, then, I mean that its referent exists. (Again, different philosophers - e.g. 'actualists' on one side, 'possibilists' on the other - may wish to interpret this 'exists' in different ways.) (We will not need to define instantiation for complex substancenotions.)

We come finally to logicality. Let $\mathrm{s}$ be any simple notion (whether attributive notion or substance-notion); we then define:

$\mathrm{s}$ is logical $=_{d f}$ Necessarily, $\mathrm{s}$ is instantiated.

Necessity here is 'metaphysical' necessity.

Now no doubt it should be the case that a complex notion is logical if and only if all the simple notions occurring in it are logical. (See BATCHELOR 2013, §§ 5-6.) So given any complex notion $\mathrm{x}$, we 
can define ' $\mathrm{x}$ is logical' as the conjunction of all propositions [ $\mathrm{s}$ is logical] (in the sense already defined) for s a simple notion occurring in $\mathrm{x}$. This completes the presently proposed definition of logicality.

It should be emphasized here that it would not be adequate to define logical notions in general (including complex ones) as necessarily instantiated notions. (Thus the title of the present note is elliptical (for the sake of lapidarity).) Indeed we have not even defined instantiation for complex substance-notions. But irrespectively of that, there are obvious counterexamples with complex attributive notions, as already indicated above in $\S 1$.

\section{Agreement with customary classification of specific notions}

Now, how far does the presently proposed definition of logicality agree with the customary classification of specific notions or classes of notions as logical or nonlogical? - Very far, it seems to me.

It will be enough to consider simple notions, or reasonable candidates for being simple notions, since the adequacy of our general definition relative to the adequacy of our definition of logicality for simple notions is straightforward.

Let us begin with what are usu- ally reckoned as being (plausibly simple) logical notions.

The usual truth-functional connectives negation, conjunction, disjunction (or the Sheffer stroke etc.) are obviously necessarily instantiated. E.g. if all men are mortal then negation is instantiated by [Not all men are mortal]; and if not all men are mortal then negation is instantiated by [All men are mortal]. Only a 'falsum' truthfunction, $\perp$, would be a counterexample; but surely it is implausible to think that there is a simple notion here; it seems much more plausible to think that $\perp$ (A) should be defined as (say) $\mathrm{A} \wedge \neg \mathrm{A}$ (and similarly for the binary, ternary etc. falsum connectives).

(Another way around an alleged simple falsum would be to re-define: $\mathrm{s}$ is logical $=_{d f}$ Either it is necessary that $s$ is instantiated or it is necessary that $\mathrm{s}$ is not instantiated - or in other words: Whether $\mathrm{s}$ is instantiated is not a contingent matter. It may also be argued on independent grounds that this is, conceptually, a more satisfactory definition: empiricality (of a simple) is here, naturally, equated to contingency of instantiation, and logicality, which is the contradictory of empiricality, is thus equated to non-contingency of instantiation. - The empirical realm is the realm of contingencies, and the logical realm the realm 
of non-contingencies. - However, it may also be argued on the other side that this (slightly more complex) reformulation is hardly worth the trouble, since surely there are no necessarily uninstantiated simple notions, so that the two formulations are in fact equivalent.)

Again, the universal and existential quantifiers, identity, metaphysical necessity and metaphysical possibility - these are all necessarily instantiated.

(For someone who thinks that there might be nothing at all, the above-suggested definition of logicality would inadequately count e.g. the existential quantifier as non-logical. But the following amended version would do, I suppose: $\mathrm{s}$ is logical $=_{d f}$ Necessarily: if there is anything at all, then $s$ is instantiated. - But now, if it is possible that there is exactly one thing, and say the quantifier [At least two things] is a simple notion, then this definition would still wrongly classify it as nonlogical. But of course it is much more plausible to think that [At least two things] is a complex notion; and the same holds for the other obvious putative counterexamples which might be given in this connection.)

Also, if there are simple notions of set-membership, empty set, etc., or of natural numbers and usual numerical relations etc., then these will count as logical by our definition, as they should, given that as I said this is meant as definition of logicality in a broad sense (or 'logicomathematicality'). (Assuming of course standard 'rigid' rather than eccentric 'contingent' Platonism.)

Coming now to simple notions usually reckoned as not being logical notions, it seems clear that they will indeed count as nonlogical by our definition. No doubt simple empirical particulars do not necessarily exist; no doubt simple empirical properties and relations of particulars are not necessarily instantiated. And there is no good reason that I know of for thinking that there are any simple empirical propositional connectives at all, let alone necessarily instantiated ones; and similarly for other supposed simple higher-order empirical notions.

(In another paper (BATCHELOR 2011), I have suggested another, quite differently motivated, definition of logicality. That definition had some consequences (explicitly indicated in that other paper, § 2) which some philosophers have found too drastic. The present definition has no such consequences, and I hope that others may find it more acceptable. As for my giving two 
different definitions of logicality, I table analysis leading to the unido not see any problem about it: que 'essence' of the idea of logithese are two different conditions cality (which would be a hopeless both of which, I conjecture, pick goal in my opinion). So there is no out precisely what should count problem if we have more than one as logical notions - no claim being such condition; it would be a promade in either case to an inevi- blem if we had less than one.)

\section{References}

BATCHELOR, R. 'Topic-Neutrality', Mind, vol. 120, 2011, pp. 1-9.

BATCHELOR, R. 'Complexes and Their Constituents', Theoria (Lund), vol. 79, 2013, pp. 326-352. 
